\title{
Design of Digital Geophone System Based on FPGA
}

\author{
Yi Chen \\ School of Electronic and Information Engineering \\ Soochow University \\ Suzhou, 215006, China \\ E-mail: chenyipure@163.com
}

\author{
Donghai Qiao \\ Institude of Acoustics \\ Chinese Academy of Science \\ Beijing, 100190, China \\ E-mail: qiaodh@suda.edu.cn
}

\begin{abstract}
In order to meet the needs of high-resolution seismic data acquisition, a kind of 24-bit high performance digital geophone was designed. The digital geophone was designed with a low-power, high-integration FPGA as the acquisition controller, and SOPC embedded system was established to control the whole collection station. This paper gives the overall architecture of the geophone and concrete design and implementation of the system's main peripheral circuits, including the sensor's self-test circuit, preamplifier filter circuit, 24-bit analog-to-digital conversion circuit and communication interface circuit. Each of these parts was optimally designed for low power. The system is controlled based on Verilog HDL and Nios II soft core. In this way, the area of circuit board is reduced efficiently, and high speed acquisition and high precision measurement can be realized. Finally, with the use of simulated seismic waves generated by the vibration table, we completed the data acquisition and transmission experiment of the acquisition system containing three collection nodes. The results show that this system is of high precision, high reliability, low power, low cost and it is easy to be upgraded and maintained.
\end{abstract}

Keywords-digital geophone; FPGA; acquisition system; 24-bit $\triangle-\Sigma A D C$

\section{INTRODUCTION}

Seismic exploration is the most reliable and commonly used technology in oil exploration. Geophone is the most important tool of seismic data acquisition, and its development directly influences the accuracy of the exploration[1]. Traditional analog geophone transfers the detected analog signal to the data acquisition station through detection wires. The detecting part and analog-digital conversion components are separated at this time. In such a seismic exploration system, bottleneck effect should be strongest in analog-signal transmission. Analog signal will be affected by various interfering factors in long-distance transmission, which will further reduce the signal to noise $\operatorname{ratio}(S N R)$, and influence the exploration accuracy[2].

Compared with analog geophone, digital geophone has advantages of high accuracy, low failure rate, light weight, small size and easy networking and it's the current development trend[3]. Its important feature is that A/D conversion is completed inside the detector, and it outputs the digital signal directly. Combine the moving-coil analog geophone with digitizing circuits, and we can get the same good results. If the analog detector is digitized, it can make up for the short of analog geophone like small line interference and transmission loss, which will greatly improve the quality of seismic data, and bring the convenience for high-density seismic exploration[4]. The digital geophone designed integrates the sensor, data acquisition and transmission circuit and power, and the analog signal can be directly converted to digital signal, then transferred to host computer, which effectively eradicates the long-distance transmission of analog signal, improves the SNR of acquisition signal, and enhances anti-jamming capability of the equipment.

\section{ARCHITECTURE OF THE GEOPHONE SYSTEM}

The digital geophone system uses Altera's Cyclone IV FPGA EP4CE22E22C6N as the core controller. Its block diagram is shown in Fig.1. It mainly includes: power supply voltage conversion circuit, preamplifier filter circuit, A/D conversion circuit, sensor's self-test circuit, FPGA core circuit, and communication interface circuit.

The number of logical units on EP4CE22E22C6N chip is up to 22320. This chip has the feature of low power and high integration, and its embedded RAM block size is $594 \mathrm{~Kb}$. Abundant logic cells and RAM number bring adequate resources for on-chip hardware design. In this design, SOPC embedded system is established to control the whole collection station, and the control logic of some peripherals is realized by verilog HDL. The digital logic circuit has the benefit of high data transmission speed, and Nios II soft-core processor based on C-language design has the advantage of strong data processing capability[5]. Therefore, the whole system performance is improved greatly, and the two different application circuit can be implemented and integrated on a

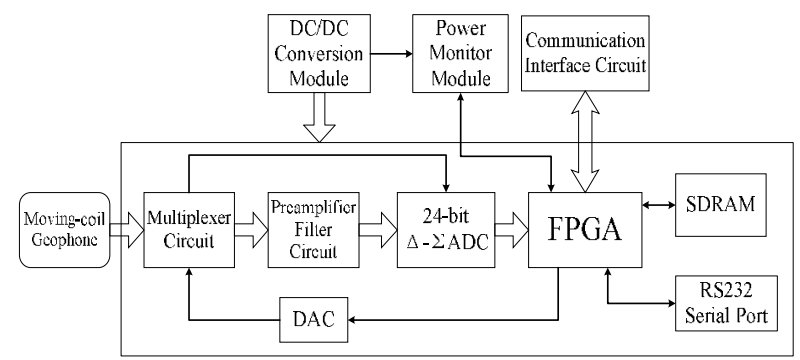

Fig.1. Block diagram of a single point digital geophone 
single chip, which greatly reduces the hardware cost, board area, and complexity of software design.

The ISSI company's synchronous dynamic random access memory IS42S81600F is connected to FPGA. This SDRAM has a memory size of $16 \mathrm{MB}$. In this system, on the one hand, it is used as the external expansion memory of Nios II soft-core processor, on the other hand, it is used for storing the collected seismic data temporarily. The geophone's self-test signals and acquired seismic wave signal are selected to the corresponding sample channel of the ADC by the multiplexer select switches, which can be easily controlled by program. The acquired data is finally sent to the host computer through the RS232 serial port, and the computer can also transmit commands to geophone system through the interface. This design uses multiple-output flyback switching power supply to implement the power voltage conversion, which improves the efficiency of power conversion and reduces the power consumption of the whole system.

\section{HARDWARE DESIGN OF MAIN UNITS}

\section{A. Self-test Circuit Design of the Geophone}

As a sensor converting the seismic signal to electrical signal, the detector is a key component of the digital geophone. Its performance is related to the function of the whole system, so it is necessary to check the geophone first before the seismic data acquisition. The principle of self-test is mainly based on the resistance of the detector[6].The self-test circuit of the geophone is shown in Fig.2. The chip LTC1669 produced by Linear is a 10-bit rail-to rail low-power DAC, which communicates with FPGA via $\mathrm{I}^{2} \mathrm{C}$ bus.

The output of the DAC controlled by the FPGA will be filtered by RC filter, then we'll get a direct-current voltage or a sine wave signal Uo. If the signal voltage goes through resistor $\mathrm{R} 1, \mathrm{R} 2$ and $\mathrm{Rb}$ composed of parallel resistors $\mathrm{R}$ and detector's resistance $r$, the voltage $\mathrm{U}_{\mathrm{AB}}$ of the $\mathrm{Rb}$ will be:

$$
U_{A B}=\frac{R_{b}}{R_{1}+R_{b}+R_{2}} \cdot U_{o}
$$

Then $\mathrm{U}_{\mathrm{AB}}$ will be converted to digital signal by $A D C$, which can be collected by FPGA. In general, the impedance of the geophone is constant, i.e., $\mathrm{r}$ is in a certain range, so the voltage data collected by FPGA is within a certain range. If the voltage data exceeds this range, we can assure that there is something wrong with the detector or the channel. The geophone is generally buried under the earh surface, some failures like break circuit, short circuit or the stuck coil spring will occur, which makes the geophone impossible to start up[6].

\section{B. Preamplifier Filter Circuit}

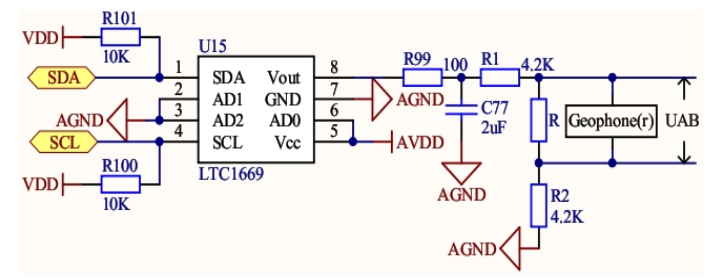

Fig.2. Self-test circuit diagram of the geophone
Seismic signal is usually very weak, in order to facilitate signal acquisition and data processing and analysis after acquisition, it's necessary for the digital geophone to amplify and filter the signal before the ADC collects the detected signal. Due to large changes in the working environment of the geophone, in order to meet the record requirements of microseismic signal in different strength, the gain of preamplifier should be designed to be programmable, so that we can ensure that the instrument has enough amplification capability and dynamic range. This design used two LMV771 amplifiers which have low drift, low noise, and rail-to-rail output swing to build a fully differential amplifier filter circuit with two optional gains. The specific circuit diagram is shown in Fig.3, wherein Rin $=$ Rin ', R18 = R5, R19 = R6. According to the system requirement, the opening and closing of the seletor switch is controlled by the program, so different amplifier gains can be selected. The signal captured by the geophone will transfer to the first channel of ADC after being amplified and filtered.

\section{A/D Converter Interface Circuit}

The system uses ADS1254 as the A/D converter, which is a 24-bit $\Delta-\Sigma$ ADC with high accuracy, low power, low noise and wide dynamic range. Its 24 significant bits can meet the seismic signal recording requirement of $120 \mathrm{~dB}$ dynamic range. Its maximum conversion rate can be up to $20.833 \mathrm{KHz}$, and there are four differential input channels and a 1-bit serial data output interface on it. The specific interface circuit is shown in Fig.4.

The normal work of ADS1254 needs three external power supplies, such as the analog power supply $\operatorname{AVDD}(+5 \mathrm{~V})$, the digital power supply $\operatorname{DVDD}(+3.3 \mathrm{~V})$, and the reference voltage $\operatorname{VREF}(+2.5 \mathrm{~V})$ for the input analog signal conversion. In order to ensure that the ADC gets higher conversion accuracy, on the one hand, the supply voltages need better filtering, and the 10 $\mathrm{uF}$ and $0.1 \mathrm{uF}$ ceramic capacitor can be placed closely to each power supply pin, on the other hand, it's necessary to make the analog ground and digital ground isolated and avoid running digital lines under the device as they may couple noise onto the

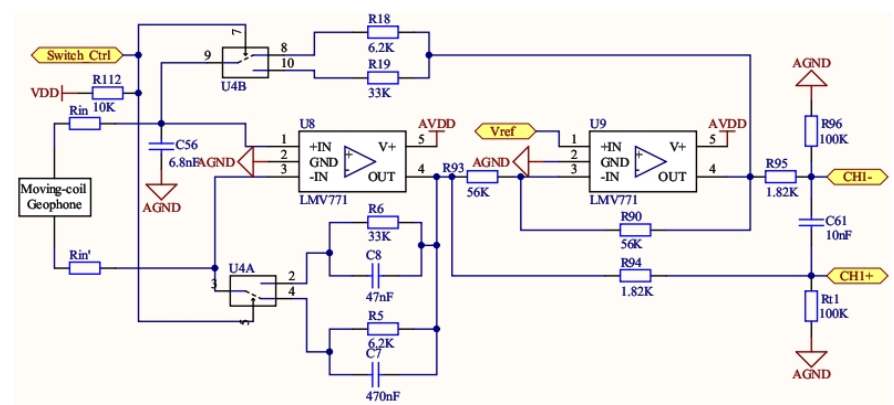

Fig.3. Preamplifier filter circuit

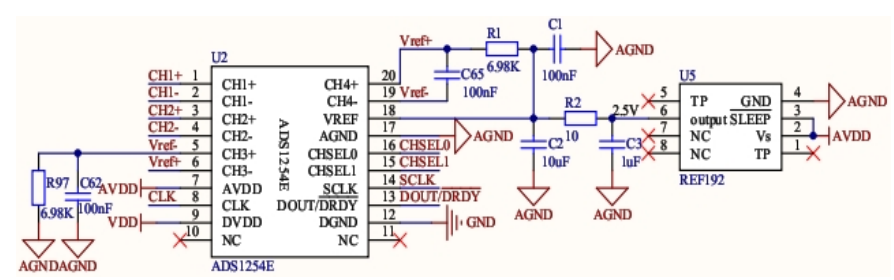

Fig.4. A/D converter interface circuit 
die. We use the precision, micropower, low-dropout-voltage, low-voltage reference REF192 to provide $+2.5 \mathrm{~V}$ reference voltage VREF for ADS1254, which can ensure the accuracy and stability of VREF.

There are four input channels on ADS1254, and the current conversion channel can be selected by controlling the voltage on the CHSEL1 and CHSEL0 pins. The input of channel $1(\mathrm{CH})$ is the generated voltage when the geophone is selftested. The input of channel $2(\mathrm{CH} 2)$ is the amplified and filtered signal acquired by the geophone. The input of channel $3(\mathrm{CH} 3)$ and channel $4(\mathrm{CH} 4)$ are the negative and positive value of the reference voltage, which is used for calibrating and compensating the reference voltage at conversion calculation. Only one channel can be selected in working state at one time.

\section{Design of Communication Circuit}

The digital geophone designed in this paper is serially connected to digital transmission line, so there are two communication ports on it, one is connected to the front stage and the other is connected to the rear stage. The circuits of two communication ports are the same, wherein one communication interface circuit is shown in Fig.5. Each communication port also has two identical signal transmission lines, both of which can send and receive signals. Signal transfers in the differential form, what's more, the signal on the geophone board and the signal in the digital transmission line are coupled by a network transformer, which ensures the electrical isolation. The chip ADCMP608 is a rail-to-rail output, fast and low-power TTL/CMOS comparator, which has a shutdown controlling pin, and should be powered by a single supply from $2.5 \mathrm{~V}$ to $5 \mathrm{~V}$. It is used to convert the differential signal received to single-ended signal for FPGA acquisition. D1 and D2 are bidirectional TVS tubes, which are used to protect the communication port from the destructive surge signal.

\section{IMPLEMENTATION AND PRINCIPLE OF THE ACQUISITION SYSTEM}

We didn't carry out the experiment in outdoor field, but only used the vibration table to generate a vibration signal to simulate the seismic waves in the laboratory. Then the signal will be captured by the digital geophone and transferred to the host computer. After the host computer receives the collected

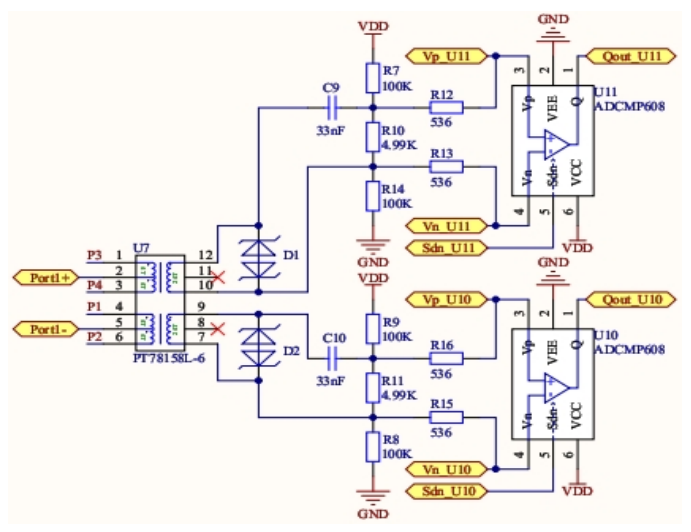

Fig.5. Communication port circuit

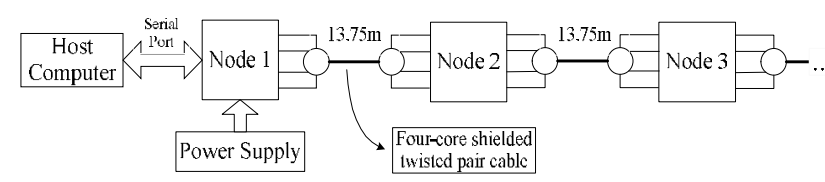

Fig.6. Block diagram of system implementation

data, we'll do further analysis. The experimental system didn't transfer every operating state of the digital geophone to the computer to display. On the digital geophone, we reserved two FPGA pins for the LED lights, so it is very convenient for debugging program and judging the executing state of each functional block through setting the LED's working state, which greatly simplifies the complexity of software design.

The system mainly implemented the serial communication among three digital geophone nodes through the RS-485 bus network. Half-duplex communication mode was used between the two nodes of them. The communication protocol of the RS485 interface in the system is UART transmission protocol, and the communication baud rate is set to $2 \mathrm{Mbps}$. Each bit of the data will be encoded before being sent by the digital geophone, and we use the encoding method similar to the Manchester encoding, which uses the rising edge that the voltage level changes from low to high to represent logic " 0 ", and the falling edge that the voltage level changes from high to low to represent logic " 1 ". The Manchester code can provide adequate timing component, and have no direct-current drift, meanwhile, the encoding and decoding process is relatively simple, so it is the best choice for seismic data transmission[7]. As shown in Fig.6, is the principle block diagram of the system implementation. Only three digital geophones are connected serially to the digital transmission line, respectively, Node 1, Node 2 and Node 3. A $13.75 \mathrm{~m}$ long standard quad twisted-pair cable is connected between them. Node 1, on the one hand, is connected to the host computer via serial cable, and responsible for the transmission and forwarding of system commands, status and the collected data, on the other hand, acts as a power station, and transfers the $+48 \mathrm{~V}$ power provided by the power supply system to the geophone string through the four-core cable. Node 2 here is only responsible for the transmission and forwarding of the system commands and the data sent from Node 3 . Node 3 mainly receives system commands and does self-testing or collects data according to the commands. After finishing collecting a certain number of data, the data will be finally transferred to Node 1 through the digital transmission lines. When Node 1 receives the data, it will do some processing and then deliver it to the host computer through the RS232 serial port.

\section{EXPERIMENTAL VERIFICATION}

The design of entire geophone system has been basically completed and verified by the board-level test. The experiment has implemented the data collection and transmission. The conversion rate of the $\mathrm{A} / \mathrm{D}$ converter $\mathrm{ADS} 1254$ was set to $20.833 \mathrm{KHz}$, which is the maximum sampling rate of the ADC and ensures the accuracy of the sampled data. The data transmission baud rate of RS-485 communication interface was set to $2 \mathrm{Mbps}$ and the data transmission was stable and reliable under test. 
First, connect the test system, and then power on the system, after the power transfers to each digital geophone, each node, on the one hand, checks the supply voltage through hardware circuit, on the other hand, conducts the DC-DC conversion of the power supply voltage to generate the required supply voltage for each module on the board. After the FPGA can start working, Nios II processor will first initialize each peripheral modules, and then waiting for the commands sent from the host computer to do corresponding processing. Node 3 will automatically complete the self-test operation after receiving the self-test command, and if no faults are detected, LED2 on board will be lighted. The connection diagram of entire experimental system and the state of Node 3 after self-test are shown in Fig.7.

After the geophone completes the self-test, set up the frequency and amplitude of the sine wave generated by the signal generator, and then connect it to the vibration table. The computer sends the signal acquisition command, Node 3 will start a data acquisition, and then automatically transmit the $32 \mathrm{~K}$ data collected to the host computer through the data transmission line. In this experiment, we collect the signal data when the signal generator outputs the sine wave whose amplitude is $250 \mathrm{mV}$, and signal frequency is $25 \mathrm{~Hz}, 75 \mathrm{~Hz}$, $125 \mathrm{~Hz}$ and $200 \mathrm{~Hz}$, respectively. Then we use Matlab to draw the waveform of the collected data shown in Fig.8. In order to display clearly, we just take the first 3000 points of each group to draw up the waveform.

From the collected data waveforms, we know that when the frequency of vibration signals is below $200 \mathrm{~Hz}$, the geophone system can well record the waveform of the vibration signals. From the amplitude of the waveform, we can also see that when the vibration frequencies are different, the sampled signal amplitude will have different responses. This is not only related to the frequency response of the moving-coil geophone, but also has a relationship with the frequency response of the vibration table.

\section{CONCLUSION}

The solution that integrates the analog geophone core, data acquisition circuit and the power supply, makes the analog seismic signals captured by the geophone directly converted to digital signal inside the geophone, which greatly improves the reliability of the collected data. However, due to the limitation of the moving-coil geophone performance, it also has some shortcomings like the harmonic distortion, being vulnerable to industrial frequency and lightning interference. Now many

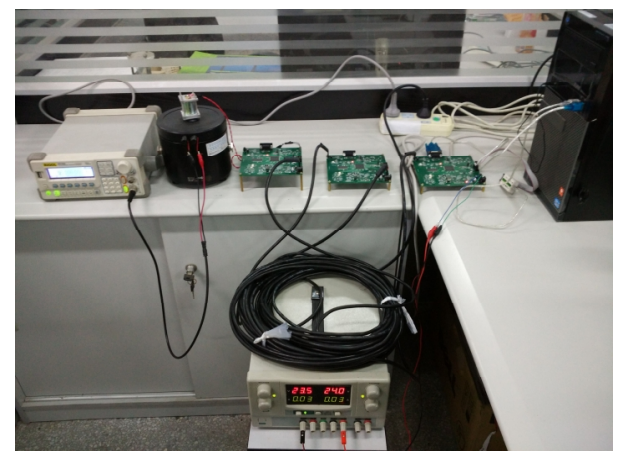

Fig.7. Connection diagram of experimental system

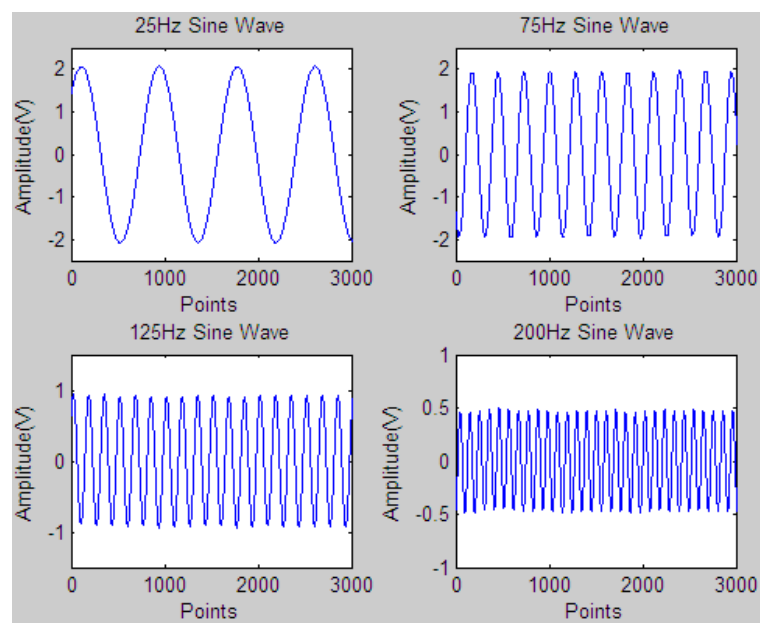

Fig.8. Sinusoidal signal waveforms of four different frequencies collected by the system

digital geophones based on MEMS acceleration sensor have been developed, which have a greater advantage in dynamic range and immunity to electromagnetic interference. However, the moving-coil geophone is more reliable and inexpensive, the requirement on the subsequent circuits is not high, circuit design is simple, and it is suitable for working in high noise situation, therefore, moving-coil geophone as a mature technology, it is not obsolete, and still in a long time, using the geophone to do seismic acquisition in the high noise terrain is a more economical option.

In a word, the proposed digital geophone solution is lowcost and low-power, and greatly reduces the difficulty in software and hardware design. It has successfully passed the software and hardware debugging, which lays a certain foundation for being used to the actual seismic exploration projects.

\section{ACKNOWLEDGMENT}

I would like to express my sincere appreciation to my supervisor, Professor Donghai Qiao, for his patient guidance and valuable comments to my thesis.

\section{REFERENCES}

[1] Shanshan Tang, Design of high-resolution A/D converter in Seismometer. Shandong, Shandong University, 2010.

[2] Guowei Zhu, Huaixiu Wang, Suping Peng, "Development and applications of a new intelligent 3-component geophone," Journal of China Coal Society, vol. 31, pp. 5-10, December 2006.

[3] Jianzhong Qi, Design of digital seismometer. Shandong, Shandong University, 2011.

[4] Zhicai Zhou, Chengye Yu, Shuqi Hou, "Analysis of digitization of the regular land geophones," EGP, vol. 22, pp.75-78, April 2012.

[5] Jianguo He, Deke Ni, Yan Zheng, FPGA circuit system design based on Nios II core, 1st ed., Beijing: Publishing House of Electronics Industry, 2010, pp. 3-4.

[6] Tengda Zhang, Yongxue Zhang, "Design of micro-seismic data acquisition system based on ADS7852," Petroleum Instruments, vol. 21, pp. 7-9, October 2007.

[7] Jun Yang, "Manchester encoding and decoding in seismic data transmission application," Electronic Component \& Device Applications, vol. 12 , pp. 72-77, 2010. 